\section{Transtornos mentais comuns e apoio social: estudo em comunidade rural da Zona da Mata de Pernambuco, Brasil}

\author{
Common mental disorders and social support \\ in a rural community in Zona da Mata, \\ Pernambuco State, Brazil
}

1 Centro de Ciências da Saúde, Universidade Federal de Pernambuco, Recife, Brasil.

Correspondência A. G. Costa Centro de Ciências da Saúde Universidade Federal de Pernambuco. Rua Manuel Dias de Morais 140, apto. 202, Salvador, BA 40155-260, Brasil. albanitagomes@msn.com

\begin{abstract}
The objective of this study was to determine the prevalence of common mental disorders and the association with social support in a community located in the Zona da Mata, a sugar cane plantation area in the State of Pernambuco, Brazil. A household survey was carried out and the SelfReporting Questionnaire (SRQ-20), the Medical Outcomes Study, and socioeconomic questions were administered to all residents over 19 years of age. Total prevalence of common mental disorders was $36.0 \%$. Multivariate logistic regression analysis showed that individuals with low social support had twice the probability of suffering from common mental disorders (OR: 2.09; 95\%CI: 1.35-3.24) as compared to those with greater support, even after adjusting for age, schooling, and work force participation. The results show the importance of investments in social support networks to promote interactions between individuals and increase individuals' self-confidence and power to deal with problems.
\end{abstract}

Mental Disorders; Somatoform Disorders; Social Support
Albanita Gomes da Costa 1

\section{Introdução}

Nos últimos tempos, tem crescido a importância atribuída a estudos de Epidemiologia Psiquiátrica pela relevância destes tanto nas decisões políticas em saúde mental quanto no direcionamento da assistência médica na atenção básica e especializada de saúde. Tais estudos têm proporcionado uma compreensão mais ampla da ocorrência e do curso dos transtor nos mentais bem como das suas conseqüências diretas e indiretas para o funcionamento individual, familiar e social da pessoa afetada 1 .

São considerados transtornos mentais comuns os transtornos somatoformes, de ansiedade e de depressão 2 ; seus sintomas são: insônia, fadiga, irritabilidade, esquecimento, difi culdade de concentração e queixas somáticas 3 . Os transtornos mentais comuns são mais freqüentes nas mulheres, nos mais velhos, nos negros e nos separados ou viúvos. Tem sido verificada também a associação dos transtornos mentais comuns com os eventos vitais produtores de estresse, com o baixo apoio social e com variáveis relativas às condições de vida e trabalho tais como baixa escolaridade 4,5 , menor número de bens duráveis 6 , condições precárias de moradia 5 , baixa renda 4,5 , desemprego e informalidade nas relações de trabalho 7,8 .

O apoio social é o suporte emocional ou prático dado pela família e/ou amigos na forma de afeto, companhia, assistência e informação, tu- 
do que faz o indivíduo sentir-se amado, estimado, cuidado, valorizado e seguro 9 . Valla 10 afirma que o apoio social contribui para a sensação de coerência da vida e o controle sobre a mesma e traz benefícios não só para quem o recebe, na forma de informação ou auxílio, mas também para quem o oferece. As pessoas necessitam umas das outras e, por isso, quando o apoio social diminui há um comprometimento do sistema de defesa do corpo 11. Para Cassell 12, a sensação de não poder controlar a própria vida, juntamente com a sensação de isolamento, podem ser relacionadas com o processo de saúde-doença, aumentando a suscetibilidade individual para as enfermidades.

De acordo com Olstad et al. 13, existem duas teorias que explicam a associação entre saúde mental e apoio social. A primeira refere-se ao fato de que o apoio social afetaria diretamente a saúde mental; a segunda, ao fato de que o apoio social funcionaria como mediador do estresse, modificando o seu efeito, ou seja, o indivíduo que contasse com alto nível de apoio social reagiria mais positivamente às situações estressantes se comparado a outros que não dispusessem deste tipo de recurso.

Este trabalho tem como objetivo estimar a prevalência dos transtornos mentais comuns e sua associação com o apoio social em moradores da comunidade do Pirauá, em Macaparana, na Zona da Mata pernambucana, Brasil.

\section{Métodos}

\section{Área de estudo}

A Zona da Mata tem sua importância econômica centrada na agroindústria da cana-de-açúcar, que envolve canaviais e engenhos, abrangendo 42 municípios; além de ter a segunda maior densidade demográfica de Pernambuco: 212 habitantes $/ \mathrm{km}^{2}$. Caracteriza-se historicamente pela concentração de terra e renda com altos níveis de pobreza e poucas oportunidades de trabalho que dependem da periodicidade da safra da cana 14.

A comunidade de Pirauá conta com 1.267 habitantes e é formada sobretudo por famílias de trabalhadores rurais da cana-de-açúcar e ou pequenos produtores, com baixo nível de escolaridade e renda 15.

\section{População de estudo e coleta de dados}

Foi realizado um estudo de corte transversal na comunidade do Pirauá, Macaparana, Pernambuco no período de dezembro de 1999 a janei- ro de 2000. Todos os maiores de 19 anos de idade foram entrevistados em seus domicílios por dez estudantes do curso de enfermagem da Universidade Federal de Pernambuco, previamente treinados para a atividade, auxiliados por quatro agentes comunitários de saúde de Pirauá.

\section{- Instrumento de coleta de dados}

O Self Reporting Questionnaire (SRQ-20), desenvolvido por Harding et al. 16, tem como finalidade identificar casos suspeitos de transtornos mentais comuns em populações sem médicos especialistas. Os indivíduos respondentes foram divididos em dois grupos, de acordo com os escores do SRQ-20: não suspeito (escore igual ou menor que cinco) e suspeito (igual ou acima de seis). Tal instrumento foi validado no Brasil com sensibilidade de $85,0 \%$ e especificidade de $80,0 \% 17$, e em Pernambuco com sensibilidade de $62,0 \%$ e especificidade de $80,0 \%$, tendo como padrão-ouro a entrevista psiquiátrica 5. O Medical Outcomes Study Questions Social Support Survey (MOS-SSS) foi desenvolvido por Sherbourne \& Stewart em 1985 e é um instrumento rápido, multidimensional, de fácil administração e compreensão 18. A confiança na sua escolha como escala de apoio social veio do fato de ele já ter sido testado e validado em português 19 , não se fazendo necessário, no presente estudo, o teste de campo de sua tradução. É composto por 19 questões, compreendendo cinco dimensões funcionais de apoio social 18: apoio emocional, afetivo, tangível (provisão de recursos práticos e ajuda material), de informação e de companhia ou interação so cial. Suas perguntas podem ser respondidas de cinco maneiras diferentes: nunca, raramente, às vezes, quase sempre, ou sempre. Embora seja um instrumento auto-respondido, em razão do baixo nível de escolaridade da população estudada, foi feita a opção de administrar o MOS-SSS por meio de entrevistas. Para cada resposta, foram atribuídos escores que variavam de 1 (nunca) a 5 (sempre), efetuando-se, em seguida, a soma dos pontos obtidos em cada pergunta. $\mathrm{O}$ valor 0 foi atribuído aos itens não respondidos. Inicialmente os indivíduos foram divididos em três categorias de apoio social global e posteriormente agrupados em: baixo apoio social ( 0 a 33 pontos) e alto apoio social (34 pontos ao máximo).

\section{- Análise dos dados}

Os dados foram digitados duas vezes por diferentes pessoas com o propósito de validação e 
analisados com a utilização do programa Stata 6.0 para Windows. O escore dicotômico do SRQ20, aqui assumido como uma aproximação quantitativa do estado de saúde mental, constitui-se na variável dependente da presente análise. Investigou-se a associação do apoio social com os transtornos mentais comuns, estimando-se os odds ratio (OR) simples, intervalos de confiança a 95\% (IC95\%) e valores de p. A regressão logística foi utilizada para analisar a independência da associação dos transtornos mentais comuns com o apoio social. As co-variáveis incluídas no modelo foram aquelas descritas na literatura como potenciais fatores de confusão e que no presente estudo mostraramse associadas com os transtornos mentais comuns e com o apoio social.

\section{Resultados}

Apenas uma pessoa recusou-se a participar do estudo que teve, além desta, mais 16 perdas por motivo de incapacidade de responder às questões (surdez, deficiência mental, outros). Uma vez contabilizadas as perdas $(3,5 \%)$, o estudo contou com 483 pesquisados.

A Tabela 1 mostra a distribuição da população por variáveis demográficas, sócio-econômicas e apoio social recebido. A idade, o número de pessoas por domicílio, a escolaridade e a participação no mercado de trabalho apresentaram associação estatisticamente significativa com o apoio social (Tabela 2).

A Tabela 3 apresenta a associação dos transtornos mentais comuns com o apoio social. As pessoas com baixo apoio social (OR: 2,23; IC95\%: 1,47-3,36) apresentaram maior prevalência de transtornos mentais comuns do que as com alto apoio social. O apoio social manteve-se associado aos transtornos mentais comuns (OR: 2,09; IC95\%: 1,35-3,24) mesmo após o ajuste por idade, escolaridade e participação no mercado de trabalho $(\mathrm{p}=0,001)$.

\section{Discussão}

Este trabalho investigou a associação entre transtornos mentais comuns e apoio social. Descreve a prevalência dos transtornos mentais comuns numa realidade bastante singular da Zona da Mata pernambucana e assinala a importância do apoio social para minimizar a prevalência da doença mental.

Algumas medidas foram adotadas para minimizar as limitações do estudo como o treinamento dos entrevistadores, realização de ques-
Tabela 1

Distribuição da amostra por variáveis demográficas, sócio-econômicas, apoio social e transtornos mentais comuns.

\begin{tabular}{|c|c|c|}
\hline Variáveis & $n=483$ & $\%$ \\
\hline \multicolumn{3}{|l|}{ Sexo } \\
\hline Homem & 200 & 41,41 \\
\hline Mulher & 283 & 58,59 \\
\hline \multicolumn{3}{|l|}{ Idade (em anos)* } \\
\hline $20-39$ & 180 & 37,58 \\
\hline $40-59$ & 173 & 36,12 \\
\hline 60 e mais & 126 & 26,30 \\
\hline \multicolumn{3}{|l|}{ Pessoas por domicílio** } \\
\hline $1-4$ & 229 & 47,51 \\
\hline $5-8$ & 213 & 44,19 \\
\hline 9 e mais & 40 & 8,30 \\
\hline \multicolumn{3}{|l|}{ Estado civil ${ }^{\star \star \star}$} \\
\hline Casado & 268 & 55,60 \\
\hline Solteiro & 150 & 31,12 \\
\hline Divorciado/separado/viúvo & 64 & 13,28 \\
\hline \multicolumn{3}{|l|}{ Presença de companheiro(a)\# } \\
\hline Sim & 339 & 70,62 \\
\hline Não & 141 & 29,38 \\
\hline \multicolumn{3}{|l|}{ Escolaridade\#\# } \\
\hline Ensino médio e mais & 61 & 12,68 \\
\hline Ensino fundamental & 222 & 46,15 \\
\hline Analfabeto & 198 & 41,16 \\
\hline \multicolumn{3}{|c|}{ Participação no mercado de trabalho\#\#\# } \\
\hline Trabalha & 222 & 46,06 \\
\hline Não trabalha & 260 & 53,94 \\
\hline \multicolumn{3}{|c|}{ Renda por pessoa do domicílio§ } \\
\hline Mais de $\mathrm{R} \$ 75,00$ & 99 & 21,66 \\
\hline Entre $\mathrm{R} \$ 50,00$ e $\mathrm{R} \$ 75,00$ & 127 & 27,79 \\
\hline Menos de $\mathrm{R} \$ 50,00$ & 231 & 50,55 \\
\hline \multicolumn{3}{|l|}{ Apoio social global§§ } \\
\hline Alto & 342 & 72,46 \\
\hline Baixo & 130 & 27,54 \\
\hline \multicolumn{3}{|l|}{ Transtornos mentais comuns } \\
\hline Negativo & 309 & 63,98 \\
\hline Positivo & 174 & 36,03 \\
\hline
\end{tabular}

Valores perdidos: * $4 ;{ }^{\star \star} 1 ;{ }^{\star \star \star} 1 ; \# 3 ; \# \# 2 ; \# \# \# 1 ; \S 26 ; \S \S 6$. 
Prevalência dos transtornos mentais comuns por variáveis demográficas e sócio-econômicas.

\begin{tabular}{|c|c|c|c|c|c|}
\hline Variável & $\mathrm{n}$ & Casos & $\%$ & OR (IC95\%) & $\mathrm{p}$ \\
\hline \multicolumn{6}{|l|}{ Sexo } \\
\hline Homem & 200 & 49 & 24,50 & 1,00 & \\
\hline Mulher & 283 & 125 & 44,17 & $2,43(1,64-3,63)$ & $<0,01$ \\
\hline \multicolumn{6}{|l|}{ Idade (em anos) } \\
\hline $20-39$ & 180 & 49 & 27,22 & 1,00 & \\
\hline $40-59$ & 173 & 75 & 43,35 & $2,05(1,31-3,19)$ & \\
\hline 60 e mais & 126 & 49 & 38,89 & $1,70(1,04-2,76)$ & $<0,01$ \\
\hline \multicolumn{6}{|l|}{ Pessoas por domicílio } \\
\hline $1-4$ & 229 & 80 & 34,93 & 1,00 & \\
\hline $5-8$ & 213 & 77 & 36,15 & $1,05(0,71-1,55)$ & \\
\hline 9 e mais & 40 & 17 & 42,50 & $1,38(0,69-2,72)$ & 0,66 \\
\hline \multicolumn{6}{|l|}{ Estado civil } \\
\hline Casado & 268 & 92 & 34,33 & 1,00 & \\
\hline Solteiro & 150 & 45 & 30,00 & $0,82(0,53-1,26)$ & \\
\hline Divorciado/separado/viúvo & 65 & 37 & 56,92 & $2,62(1,50-4,57)$ & $<0,01$ \\
\hline \multicolumn{6}{|l|}{ Presença de companheiro } \\
\hline Sim & 339 & 116 & 34,22 & 1,00 & \\
\hline Não & 141 & 58 & 41,13 & $1,34(0,90-2,01)$ & 0,15 \\
\hline \multicolumn{6}{|l|}{ Escolaridade } \\
\hline Ensino médio e mais & 61 & 12 & 19,67 & 1,00 & \\
\hline Ensino fundamental & 222 & 82 & 36,94 & $2,39(1,20-4,76)$ & \\
\hline Analfabeto & 198 & 79 & 39,90 & $2,71(1,35-5,41)$ & 0,01 \\
\hline \multicolumn{6}{|c|}{ Participação no mercado de trabalho } \\
\hline Trabalha & 222 & 60 & 26,91 & 1,00 & \\
\hline Não trabalha & 260 & 114 & 43,85 & $2,12(1,44-3,11)$ & $<0,01$ \\
\hline \multicolumn{6}{|l|}{ Renda por pessoa do domicílio } \\
\hline Mais de $\mathrm{R} \$ 75,00$ & 121 & 33 & 27,27 & 1,00 & \\
\hline Entre $R \$ 50,00$ e $R \$ 75,00$ & 105 & 37 & 35,24 & $1,45(0,82-2,56)$ & \\
\hline Menos de $\mathrm{R} \$ 50,00$ & 231 & 98 & 42,42 & $1,96(1,21-3,16)$ & 0,01 \\
\hline
\end{tabular}

$\mathrm{OR}=$ odds ratio

tionário piloto, entrevista domiciliar em todos os adultos residentes no distrito, checagem de algumas questões passíveis de erro de informação e análise multivariada.

A prevalência dos transtornos mentais comuns foi de $36,0 \%$, estando próxima à apresentada para áreas urbanas e considerando estudo realizado em Olinda, Pernambuco, $35,0 \% 5$, o que nos leva a pensar que a vida no meio rural não é tão saudável quando estão presentes fatores como a pobreza, a baixa escolaridade, a falta de trabalho dentre outros. As mulheres, os com idade entre 40 e 59 anos, os analfabetos, os que têm renda por pessoa do domicílio abaixo de $\mathrm{R} \$ 50,00$ por mês e os divorciados, separados ou viúvos apresentam maior chance de ter transtornos mentais comuns. Este resultado concorda com o que foi apresentado por Lima 1 , ainda que em populações marcadas por diferenças regionais como o Pernambuco e o Rio Grande do Sul. Com relação à quantidade de pessoas por domicílio, este estudo não encontrou associação de significância estatística com a prevalência dos transtornos mentais comuns, situação que pode ter ocorrido porque, se por um lado, dividir o espaço físico com muitas pessoas pode significar um maior apoio social, por outro, pode também representar uma 
redução da privacidade que, em longo prazo, seria um fator estressante.

Estudos referidos na introdução deste trabalho mostram que o apoio social habilita o indivíduo a lidar com os eventos e condições estressoras funcionando, desta forma, como agente protetor contra os transtornos mentais comuns. Duas hipóteses tentam explicar a ação do apoio social: uma assinala um efeito de interatividade entre o apoio e os diferentes níveis de estresse em que o primeiro minimizaria o segundo (buffering hypothesis); outra (positive effects hypothesis) relata que relacionamentos provedores de apoio promovem bem-estar mesmo na ausência de estresse 20.

Saber-se amado e cuidado e ter amigos íntimos e confidentes está relacionado a baixos níveis de ansiedade, depressão e somatizações, melhor adaptação a circunstâncias particulares de estresse e menores efeitos dos eventos vitais produtores de estresse que, embora não possam ser evitados, passam a ter menores conseqüências.

É possível entender a importância do apoio social na vida dos moradores da Zona da Mata pernambucana que convivem com tamanhas dificuldades. A comunidade estudada é um distrito separado por $12 \mathrm{~km}$ da sede da cidade, com apenas oito ruas de ladeiras cujo transporte ligando ao centro não é oferecido à noite, com poucas opções de lazer e ocupação, onde as pessoas vivem sem maiores perspectivas de sucesso profissional e pessoal, caracterizado pelo isolamento e distanciamento de outras realidades. Em um lugar assim, tudo contribui para o surgimento de transtornos de ansiedade, depressão e somatizações. A solução é a integração com outras pessoas para vencer as dificuldades cotidianas e íntimas. Assim sendo, quem não pode contar com o apoio social fica ainda mais suscetível aos transtornos mentais comuns.
Tabela 3

Associação do apoio social com variáveis demográficas e sócio-econômicas.

\begin{tabular}{|c|c|c|}
\hline \multirow[t]{2}{*}{ Variável } & \multicolumn{2}{|c|}{ Apoio social } \\
\hline & Alto $(\%)$ & Baixo (\%) \\
\hline \multicolumn{3}{|l|}{ Sexo } \\
\hline Homem & $142(73,20)$ & $52(26,80)$ \\
\hline Mulher & $200(71,94)$ & $78(28,06)$ \\
\hline \multicolumn{3}{|l|}{$p=0,764$} \\
\hline \multicolumn{3}{|l|}{ Idade (em anos) } \\
\hline $20-39$ & $144(80,00)$ & $36(20,00)$ \\
\hline $40-59$ & $128(75,74)$ & $41(24,26)$ \\
\hline 60 e mais & $68(57,14)$ & $51(42,86)$ \\
\hline \multicolumn{3}{|l|}{$p<0,01$} \\
\hline \multicolumn{3}{|l|}{ Pessoas por domicílio } \\
\hline $1-4$ & $161(72,20)$ & $62(27,80)$ \\
\hline $5-8$ & $159(76,08)$ & $50(23,92)$ \\
\hline 9 e mais & $22(56,41)$ & $17(43,59)$ \\
\hline \multicolumn{3}{|l|}{$p=0,04$} \\
\hline \multicolumn{3}{|l|}{ Estado civil } \\
\hline Casado & $194(73,76)$ & $69(26,24)$ \\
\hline Solteiro & $107(73,79)$ & $38(26,21)$ \\
\hline Divorciado/separado/viúvo & $41(64,06)$ & $23(35,94)$ \\
\hline$p=0,271$ & & \\
\hline \multicolumn{3}{|l|}{ Presença de companheiro } \\
\hline Sim & $241(72,81)$ & $90(27,19)$ \\
\hline Não & $100(71,94)$ & $39(28,06)$ \\
\hline \multicolumn{3}{|l|}{$p=0,848$} \\
\hline \multicolumn{3}{|l|}{ Escolaridade } \\
\hline Ensino médio e mais & $54(88,52)$ & $7(11,48)$ \\
\hline Ensino fundamental & $170(76,92)$ & $51(23,08)$ \\
\hline Analfabeto & $117(61,90)$ & $72(38,10)$ \\
\hline \multicolumn{3}{|l|}{$p<0,01$} \\
\hline \multicolumn{3}{|c|}{ Participação no mercado de trabalho } \\
\hline Trabalha & $173(79,36)$ & $45(20,64)$ \\
\hline Não trabalha & $169(66,54)$ & $85(33,46)$ \\
\hline \multicolumn{3}{|l|}{$p<0,01$} \\
\hline \multicolumn{3}{|l|}{ Renda por pessoa do domicílio } \\
\hline Mais de $R \$ 75,00$ & $90(75,63)$ & $29(24,37)$ \\
\hline Entre $\mathrm{R} \$ 50,00$ e $\mathrm{R} \$ 75,00$ & $75(73,53)$ & $27(26,47)$ \\
\hline Menos de $\mathrm{R} \$ 50,00$ & $162(71,68)$ & $64(28,32)$ \\
\hline$p=0,730$ & & \\
\hline
\end{tabular}

Prevalência dos transtornos mentais comuns segundo apoio social, odds ratios (OR) não ajustados e ajustados e intervalos de confiança de 95\% (IC95\%).

\begin{tabular}{|c|c|c|c|c|c|c|}
\hline Variável & Prevalência (\%) & OR1 & OR2 & OR3 & OR4 & OR5 \\
\hline \multicolumn{7}{|c|}{ Apoio social } \\
\hline Alto & 30,99 & 1,00 & 1,00 & 1,00 & 1,00 & 1,00 \\
\hline Baixo & 50,00 & $2,23(1,47-3,36)$ & $2,25(1,47-3,50)$ & $2,08(1,36-3,17)$ & $2,03(1,33-3,09)$ & $2,09(1,35-3,24)$ \\
\hline Valor de $p$ & $<0,0001$ & $<0,0001$ & $<0,001$ & $<0,001$ & $<00,01$ & 0,001 \\
\hline
\end{tabular}

OR1 = simples; OR2 = ajustado por idade; OR3 = ajustado por escolaridade; OR4 = ajustado por participação

no mercado de trabalho; OR5 = ajustado por todas as variáveis. 
Valla 10, Minkler 11 e Cassell 12 defendem que a sensação de não poder controlar a própria vida e o isolamento influem maleficamente na saúde e que o empowerment, isto é, o processo pelo qual os indivíduos em grupos sociais e organizações passam a ter mais controle sobre seus destinos, traria melhorias para a saúde física, mental e emocional.

\section{Resumo}

O objetivo deste trabalho foi determinar a prevalência dos transtornos mentais comuns e sua associação com o apoio social em uma comunidade da Zona da Mata de Pernambuco, Brasil. Foi conduzido um inquérito domiciliar e o Self Reporting Questionnaire (SRQ-20), $o$ Medical Outcome Study Question e questões sobre as condições sócio-econômicas foram aplicados à população residente maior de 19 anos. A prevalência total dos transtornos mentais comuns foi de 36,0\%; a análise multivariada utilizando a regressão logística demonstrou que as pessoas que têm baixo apoio social apresentam duas vezes mais chances de ter transtornos mentais comuns (OR: 2,09; IC95\%: 1,35-3,24) que os que têm alto apoio mesmo depois do ajuste por idade, escolaridade e participação no mercado de trabalho. Os resultados indicam a importância de se investir em redes de apoio social para promover a interação dos indivíduos e aumentar a confiança pessoal e o poder de enfrentamento dos problemas.

Transtornos Mentais; Transtornos Somatoformes; Apoio Social
Isso posto, é importante investir em redes de apoio social com a criação de grupos de "cuidado social" em que o indivíduo receba informação sobre os mais diversos assuntos e tenha atividades recreativas que promovam interação com os demais membros e priorize o envolvimento comunitário para aumentar a confiança pessoal, a satisfação com a vida e o poder de enfrentamento dos problemas.

\section{Colaboradores}

A. G. Costa participou na redação do texto e elaboração das tabelas. A. B. Ludermir colaborou com a adequação do artigo. 


\section{Referências}

1. Lima MS. Epidemiologia e impacto social. Rev Bras Psiquiatr 1999; 21 Suppl 1:1-5.

2. Mari JJ, Jorge MR. Transtornos psiquiátricos na clínica geral. Psychiatry On-line Brazil 1997; 2. http:// www.polbr.med.br/arquivo/tpqcm.htm (acessado em 14/Jun/2004).

3. Goldberg D, Huxley P. Commom mental disorders: a bio-social model. London: Tavistock; 1992.

4. Lima MS, Béria JU, Tomasi E, Conceição AT, Mari JJ. Stressful life events and minor psychiatric disorders: an estimate of the population attributable fraction in a Brazilian community-based study. Int J Psychiatry Med 1996; 26:211-22.

5. Ludermir AB, Melo Filho DA. Condições de vida e estrutura ocupacional associadas a transtornos mentais comuns. Rev Saúde Pública 2002; 36:21321.

6. Power C, Mannor O, Fox J. Health and class: the early years. London: Chapman and Hall; 1991.

7. Santana VS, Loomis DP, Newman B, Harlow SD. Informal jobs: another occupational hazard for woman's mental health? Int J Epidemiol 1997; 26: 1236-42.

8. Ludermir AB, Lewis G. Informal work and common mental disorders. Soc Psychiatry Psychiatr Epidemiol 2003; 38:485-9.

9. Communities Count. Basic needs and social determinants of well being: social support. http:// www.communitiescount.org/B_social_support.h tm (acessado em 14/Jun/2002).

10. Valla VV. Educação popular, saúde comunitária e apoio social numa conjuntura de globalização. Cad Saúde Pública 1999; 15 Suppl 2:7-14.

11. Minkler M. Building supportive ties and sense of community among the inner-city elderly: the Tenderloin Senior Outreach Project. Health Educ Q 1985; 12:303-14.

12. Cassell J. Psychosocial processes and "stress": theoretical formulation. Int J Health Serv 1974; 4:471-82.
13. Olstad R, Sexton H, Sogaard AJ. The Finnmark study: social support, social network and mental distress in a prospective population study. Soc Psychiatry Psychiatr Epidemiol 1999; 34: 519-25.

14. Hamasaki CS. O setor sucroalcooleiro e seus trabalhadores: emprego e pobreza na Zona da Mata de Pernambuco [Dissertação de Mestrado]. Recife: Programa de Pós-graduação em Economia Universidade Federal de Pernambuco; 1997.

15. Silva AC, Oliveira JG. O processo participativo nas associações de moradores de Pirauá e de UrucuMacaparana/PE [Monografia]. Recife: Centro de Ciências Sociais Aplicadas, Universidade Federal de Pernambuco; 1999.

16. Harding TW, Arango MV, Baltazar J, Climent CE, Ibrahim HHA, Ladrido-Inacio L, et al. Mental disorders in primary health care: a study of the frequency and disgnosis in four developing countries. Psychol Med 1980; 10:231-41.

17. Mari JJ, Williams PA. A validity study of a Psychhiatric Screening Questionnaire (SRQ 20) in primary care in city of São Paulo. Br J Psychiatry 1996; 148:23-6.

18. Sherbourne CD, Stewart AL. The MOS social support survey. Soc Sci Med 1991; 32:705-14.

19. Chor D, Griep RH, Lopes CS, Faerstein E. Medidas de rede e apoio social no Estudo Pró-Saúde: pré-testes e estudo piloto. Cad Saúde Pública 2001; 17:887-96.

20. Sherman AM. Social relations and depressive symptoms in older adults with knee osteoarthritis. Soc Sci Med 2003; 56:247-57.

Recebido em 02/Fev/2004

Versão final apresentada em 02/Ago/2004

Aprovado em 25/Ago/2004 